\title{
ON CONSTANTS IN COCONVEX APPROXIMATION OF PERIODIC FUNCTIONS
}

\author{
GERMAN DZYUBENKO
}

Abstract. Let $2 \pi$-periodic function $f \in \mathbb{C}$ change its convexity finitely even many times, in the period. We are interested in estimating the degree of approximation of $f$ by trigonometric polynomials which are coconvex with it, namely, polynomials that change their convexity exactly at the points where $f$ does. We list established Jackson-type estimates of such approximation where the constants involved depend on the location of the points of change of convexity and show that this dependence is essential by constructing a counterexample.

Mathematics subject classification (2010): 42A10, 41A17, 41A25, 41A29.

Keywords and phrases: Coconvex approximation by trigonometric polynomials, Jackson estimates, counterexample.

\section{REFERENCES}

[1] N. I. AKHIEZER, Lectures on Approximation Theory, Moscow: Nauka, 1965 (in Russian).

[2] S. N. Bernstain, Sur la limitation des dérivées des polynomes, Compte rendus, Paris 190 (1930), 338-341.

[3] R. A. DeVore, D. Leviatan and I. A. Shevchuk, Approximation of monotone functions: A counter example, Proceedings Curves and surfaces with applications in CAGD (Chamonix-MontBlanc, 1996), Nashville, TN: Vanderbilt Univ. Press, 1997, 95-102.

[4] G. A. Dzyubenko, J. Gilewicz and I. A. Shevchuk, Coconvex pointwise approximation, Ukr. Mat. Zh., 54 (2002), 1, 1200-1212. English transl. in Ukrainian Math. J. 54 (2002), 1445-1461.

[5] G. A. Dzyubenko, J. Gilewicz and I. A. Shevchuk, New phenomena in coconvex approximation, Analysis Mathematica, 32 (2006), 113-121.

[6] G. A. Dzyubenko, D. Leviatan And I. A. Shevchuk, Nikolskii-type estimates for coconvex approximation of functions with one inflection point, Jaen J. Approx., 2 (2010), 1, 51-64.

[7] G. A. Dzyubenko, D. Leviatan, And I. A. Shevchuk, Coconvex pointwise approximation, Rendiconti del circolo matematico di Palermo, Serie II, Suppl. 82 (2010), 359-374.

[8] G. A. Dzyubenko, D. Leviatan and I. A. Shevchuk, Pointwise estimates of coconvex approximation, Jaen J. Approx., 6 (2014), 2, 261-295.

[9] G. A. DZyubenko And V. D. Zalizko, Coconvex approximation of functions that have more than one inflection point, Ukr. Mat. Zh., 56 (2004), 3, 352-365. English transl. in Ukrainean Math. J. 56 (2004), 427-445.

[10] V. K. DZYADYK, Introduction to the theory of uniform approximation of functions by polynomials, Moscow: Nauka, 1977, 512 pp. (in Russian).

[11] J. Gilewicz, Yu. V. KRYAKIN AND I. A. SheVchuk, Boundedness by 3 of the Whitney interpolation constant, J. Approx. Theory, 119 (2002), 271-290.

[12] D. JACKSON, Üeber die Genauigkeit der Annäherung stetiger Funktionen durch ganze rationale Funktionen gegebenen Grades und trigonometrische Summen gegebener Ordnung, Göttingen (1911) (Thesis).

[13] D. JACKSON, On approximation by trigonometric sums and polynomials, Trans. Amer. Math. Soc., 13 (1912), 491-515.

[14] K. A. Kopotun, D. Leviatan And I. A. Shevchuk, The degree of coconvex polynomial approximation, Proc. Amer. Math. Soc. 127 (1999), 409-415. 
[15] K. A. Kopotun, D. Leviatan, A. Prymak And I. A. Shevchuk, Uniform and pointwise shape preserving approximation by algebraic polynomials, Surveys in Approximation Theory, 6 (2011), 2474.

[16] D. Leviatan And I. A. Shevchuk, Coconvex approximation, J. Approx. Theory, 118 (2002), 20 65.

[17] G. G. LorentZ And K. L. Zeller, Degree of Approximation by Monotone Polynomials I, J. Approx. Theory, 1 (1968), 501-504.

[18] G. G. LoREnTZ AND K. L. Zeller, Degree of approximation by monotone polynomials II, J. Approx. Theory, 2 (1969), 265-269.

[19] P. A. Popov, An analog of the Jackson inequality for coconvex approximation of periodic functions, Ukr. Mat. Zh., 53 (2001), 919-928. English transl. in Ukrainean Math. J. 53 (2001), 1093-1105.

[20] P. A. Popov, One counterexample in coconvex approximation of periodic functions, - iv: Collection of works of Inst. of math. NAS of Ukraine. 2002, 35, 233 pp., 113-118 (in Ukrainian).

[21] A. S. SHVEdov, Orders of coapproximation of functions by algebraic polynomials, Mat. Zametki, 29 (1981), 1, 117-130. English transl. in Math. Notes 29 (1981), 63-70.

[22] S. B. STEChKIn, On the order of best approximations of continuous functions, Izv. USSR Academy of Sciences. Ser. mat., 15 (1951), No. 3, 219-242 (in Russian).

[23] H. Whitney, On Functions with Bouded n-th Differences, J. Math. Pures Appl. 36 (1957), 9, 67-95.

[24] X. WU AND S. P. Zhou, A counterexample in comonotone approximation in $L^{p}$ space, Colloq. Math., 64 (1993), 2, 265-274.

[25] V. D. ZaLizko, Coconvex approximation of periodic functions, Ukr. Mat. Zh., 59 (2007), 1, 29-42. English transl. in Ukrainian Math. J. 59 (2007), 28-44.

[26] V. D. ZALIZKo, A counterexample for coconvex approximation of periodic functions, - iv: Collection of scientific articles: M. P. Dragomanov Nat. ped. univ., Series 1. Physical and mathematical sciences, 2006, 6, 91-96 (in Ukrainian).

[27] S. P. ZHou, On comonotone approximation by polynomials in $L^{p}$ space, Analysis, 13 (1993), 363376.

[28] A. Zygmund, Smooth functions, Duke Math. Journal, 12 (1945), 1, 47-76. 